\title{
Hardware Design and Implementation of Optoelectronic Pod Control System Based on DSP
}

\author{
Zhigang Feng and Ming Jin \\ School of Automation, Shenyang Aerospace University, Shenyang, Liaoning, \\ China \\ fzg1023@yeah.net
}

\begin{abstract}
The optoelectronic pod always works in complicated environment, so it was impacted inevitably on various of elements such as Windage, Mechanical vibration, Load disturbance and so on. These random factors and nonlinear factors led to reduce the control precision, so much so that it can damage the hard-system of the optoelectronic pod. Traditional control system of optoelectronic pod always adopts PID control algorithm to eliminate errors between control target and actual feedback. However, the traditional PID can't track variational variables such in the complicated environment. It can led to lower the control precision and slow the speed of response. Self-adaption control system of optoelectronic pod adopts active disturbance rejection control (ADRC) technique which can track the mutational disturbance, estimates it and compensates it. In this paper, optoelectronic pod control system is designed and implemented by using TMS320F28335 to acquire the sensor signals, execute control algorithm, and drive the DC torque motor. The angular displacement sensors acquire the attitude angular displacement and the gyroscope acquires the attitude angular speed. The system can get attitude information of the optoelectronic pod with them. The motor diverters detect the driven current of motor to complete control feedback of DC torque motor. The EEPROM stores control parameters and sends relevant parameters according to DSP instructions. Experimental results indicate that DSP data processing unit can acquire the inner sensors data correctly in normal state, and perform control algorithms steadily in the disturbed environment.
\end{abstract}

Keywords: optoelectronic pod; ADRC; DC torque motor; PID;DSP

\section{Introduction}

Airborne electro-optoelectronic pod is largely applied in all fields (including investigation, collimation, monitoring, surveying, navigation, rescue, patrolling, aerial camera and etc...). Airborne electro-optoelectronic pod is divided into two categories based on its load. The first one is equipped with Photoelectron imaging system. The other is equipped with electro-optical targeting system. In some cases, Photoelectron imaging system and electro-optical targeting system are both installed in an airborne electro-optoelectronic pod to achieve the function of investigation and collimation.

The development of optoelectronic pod can be traced back to 1970s, optoelectronic pods mainly using cylindrical shaped structure early. The old-fashioned optoelectronic pod control system was set up with analogous circuits which contain many disadvantages, and mainly reflected in these aspects: low control accuracy, low level of integration, bad reliability, no self-inspection system and expensive price. In the 1990s, with the growing of optoelectronic pod applied value, many countries started to develop optoelectronic pod control system (including America, Israel, Sweden, England, French, and etc...). With the technology development, all kinds of advanced optical equipments were developed so it provides conditions for upgrading optoelectronic pod. Some countries have been in the 
world advanced level (including America, Israel, Canada, French, Russia and etc...). They have developed many types of optoelectronic pods and largely equipped them for military. The new generation optoelectronic pods both adopt digital processing units and high integration density electronic chips to reduce bulk of pod. Control precision of optoelectronic pod has already reached 0.5 urad. With these designs, the costing of optoelectronic pods had been largely decreased and application areas of pod have been enlarged. Lockheed Martin F-16 PANTERA pod is an advanced airborne electro-optoelectronic pod. PANTERA incorporates a high-resolution 3-5 $\mu \mathrm{m}$ third generation forward-looking infra-red (FLIR), ahigh-resolution near-infra-red CCD daylight TV camera, an infra-red pointer, a dual-mode laser, and a laser spot tracker.

With the development of aviation industry, the modern fighter aircraft has capability to cruise in the complex working conditions, so it brings a challenge for optoelectronic pod. The traditional control system of optoelectronic pod always adopts Proportional-integral-derivative (PID) control algorithm. PID control provides an efficient solution to control problems and make good performance in static precision [1].System input is always step value (break value) and the output is slow variety, so it is impossible that slow variety can track break value. As differentiator of PID control is not realized in the physical layer, so PID control algorithm has to adopt approximate differential method. The method not only causes low accuracy but also impacted easily on noise. The integration element of PID control may lead to slowing system response and can't compensate the high frequency disturbance. The fuzzy controller has good robust and is effective for the nonlinear time-varying system. Based on Advanced Scale Factor and Smooth Handover, Fuzzy PID Composite controller (A-FPID) can achieve high performance in static precision and dynamic characteristic for stabilized platform [1]. However, Fuzzy PID control algorithm may produce a large overshoot in the implementation process. In the engineering, system engineers design the fuzzy rules based on their design experience, so the fuzzy rule leaks will appear inevitably in the extreme environment. Optoelectronic pods hang on the moving vehicle. The vibration of carrier and wind resistance result in the instability of optical sensors and jitter of obtained video $[2,3]$.Specially, the vibration of carrier becomes unpredictable when the air force enter into low-level suddenly. To reduce the airborne vibration influence of optoelectronic pod on quality and stability, the irrotational displacement vibration isolation device was designed according to the parallelogram principle, vibration isolation theory and the analysis data of pod modal [4]. According to the need of reconnaissance mission, modern aircraft has to adopt low altitude fight, in this case, it generates severe vibration emerge inevitably. This vibration isolation device can't isolate completely vibrations, therefore, the disturbance compensation should be added to maintain dynamic stability of control system. In this paper, active disturbance rejection control (ADRC) application in the optoelectronic pod for disturbance rejection. Firstly, model of stabilized platform system is established, in which uncertain external load disturbance and unmodelled dynamics are regarded as one integrated disturbance. Then, using ESO to observe and compensate this integrated disturbance, and using the compensation loop of ADRC as the inner loop of traditional PID controller [5]. The Digital Signal Processor (DSP) operates efficiently ADRC algorithm. It can improve control performance and anti-disturbance ability for optoelectronic pod in the complex controlled environment. In this paper, the electric current loop is added to protect hard-system of airborne optoelectronic from locked-rotor current in the extreme environment.

The paper is organized as follows. In the section 2, airborne electro-optoelectronic pod system model analysis is briefly summarized. The DC torque motor model is proposed to calculate its transfer function. According its orders, the extended state observer is built to estimate system disturbance. In the section 3, constructs system framework according to control requirement, and expounds module functions in detail. In the section 4, introduces the hard-system analyzes module circuits of system in detail. In the section 5, introduces 
software design in detail and illustrates program flow chat. In the section 6 , shows some test results of the stabilization accuracy to expound that new generation of Control system which adopts active disturbance rejection control technique can maintain control precision required in each frequency.

\section{Airborne Electro-optoelectronic pod System Model Analysis}

External Disturbance mainly effects on the DC torque motors of optoelectronic pod when optoelectronic pod work in the severe environment. In order to surmount external disturbance and improve dynamic performance and stable precision of the system, analytical model of DC torque motor is required. In this paper, the DC torque motor is selected to drive optoelectronic pod in the direction of pitching and yaw. The DC torque motor can drive load directly and adjust revolving speed of load by the input control signal, meanwhile, DC torque motor can work normally in the state of lock-rotor. The selected DC torque motor parameters as follow:

Table 1. Torque Motor Parameters

\begin{tabular}{|c|c|c|}
\hline DC motor torque parameters & Unit & Value \\
\hline Peak torque at locked-rotor & $M_{f d}(N \cdot m)$ & 20 \\
\hline Peak current at peak torque & $I_{f d}(A)$ & 11.4 \\
\hline Peak voltage at locked-rotor & $U_{a}(V)$ & $27 \pm 3$ \\
\hline Racing speed at 27V & $\Omega_{0}(r p m)$ & $120 \pm 12$ \\
\hline Voltage at continuous torque & $U_{C}(V)$ & $16.7 \pm 1.7$ \\
\hline Current at continuous torque & $I_{c}(A)$ & 7 \\
\hline Continuous lock-rotor torque & $M_{c}(N \cdot m)$ & 12.5 \\
\hline Rotational inertia of rotor & $J_{M}\left(k g \cdot m^{2}\right)$ & 0.36 \\
\hline Armature resistance & $R_{a}(\Omega)$ & 2.37 \\
\hline Armature inductance & $L_{a}(m H)$ & 14 \\
\hline Ripple torque coefficient & & $\leq 15 \%$ \\
\hline
\end{tabular}

The basic principle of DC torque motor is same with DC motor, the equivalent circuit diagram as follow:

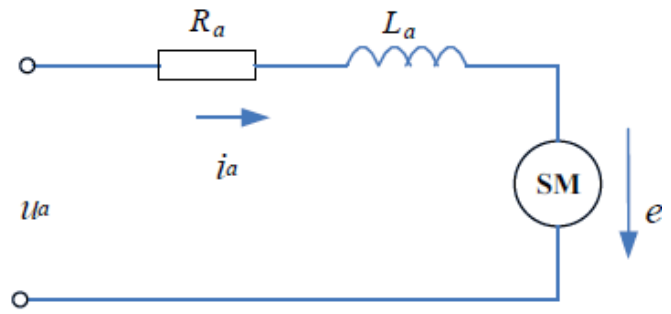

Figure 1. The Equivalent Circuit Diagram of DC Torque Motor

The armature circuit equation is given based on Kirchhoff Voltage Law(KVL)

$u_{a}=L_{a} \frac{d i_{a}}{d_{t}}+i_{a} R_{a}+e$

Where ${ }^{u_{a}}$ is input voltage, $L_{a}$ is armature inductance, $R_{a}$ is armature resistance, e is represents Back-EMF, ${ }^{i}{ }_{\text {is }}$ armature current. 
Moment equilibrium equation

$T=J \frac{d_{\omega}}{d_{t}}+T_{f}$

$T=K_{t} \omega$

Where $T$ is armature torque, $T_{f}$ is torque disturbance, $J$ is rotation inertia, $K_{t}$ is torque sensitivity coefficient, $\omega$ is motor speed.

Back Electromotive Force equation

$u_{e}=K_{e} \omega$

Where ${ }^{u_{e}}$ is back electromotive force, $K_{e}$ is back-EMF coefficient, $\omega$ is motor speed.

The block diagram of torque close-loop system is shown in figure 2:

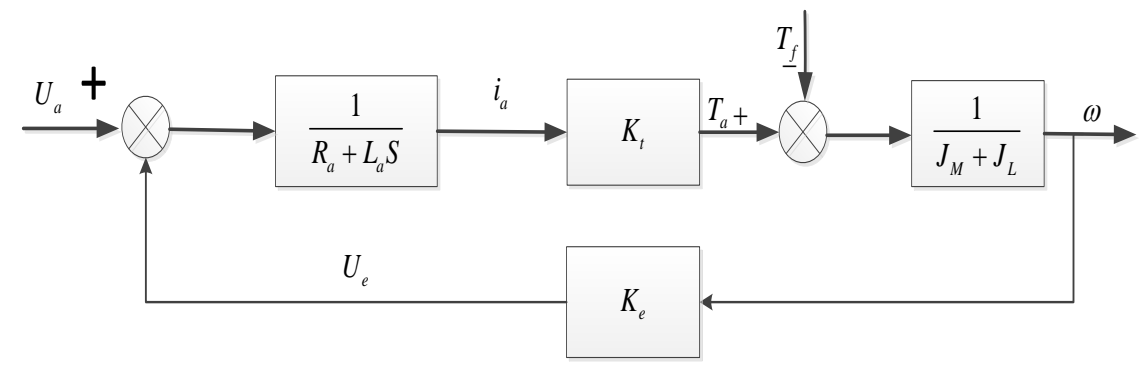

Figure 2. The Transfer Function Model

Transfer function of DC torque motor as show (2.5):

$\frac{\omega(s)}{u_{a}(s)}=\frac{\frac{1}{K_{e}}}{\frac{R_{a}\left(J_{M}+J_{L}\right)}{K_{e} K_{t}} \bullet \frac{L_{a}}{R_{a}} s^{2}+\frac{R_{a}\left(J_{L}+J_{M}\right)}{K_{e} K_{t}} s+1}=\frac{\frac{1}{K_{e}}}{T_{m} T_{e} S^{2}+T_{m} s+1}$

(

Where $T_{e}$ is electrical time constant, $T_{m}$ is motor electromechanical time constant, $K_{e}$ motor back-EMF coefficient.

According to DC motor torque parameters, parameters of transfer function are show as follow:

Motor Back-EMF coefficient:

$K_{e=} \frac{U_{a}}{\Omega_{0}}=2.15 \mathrm{~V} / \mathrm{rad} / \mathrm{s}$

$K_{t}=\frac{M_{f d}}{I_{f d}}=1.754 N \bullet m / A$

Motor Electromagnetic Constants:

$T_{e}=\frac{L_{a}}{R_{a}} \approx 0.006 \mathrm{~s}$

Motor Electromechanical Time Constant:

$T_{m}=\frac{R_{a}\left(J_{M}+J_{L}\right)}{K_{e} K_{t}}$

$T_{m} \quad=\quad 9$

The transfer function of DC torque motor is given as 
$G_{\mathrm{p}}(s)=\frac{\frac{1}{K_{b}}}{T_{m} T_{e} s^{2}+T_{m} s+1} \approx \frac{\frac{1}{K_{b}}}{\left(T_{m} s+1\right)\left(T_{e} s+1\right)}=\frac{0.465}{(24.99 s+1)(0.006 s+1)}$

1

According to formula (6), the controlled object is second order system so ADRC is designed as a second order, the structure of the second order ADRC as show in figure 3.

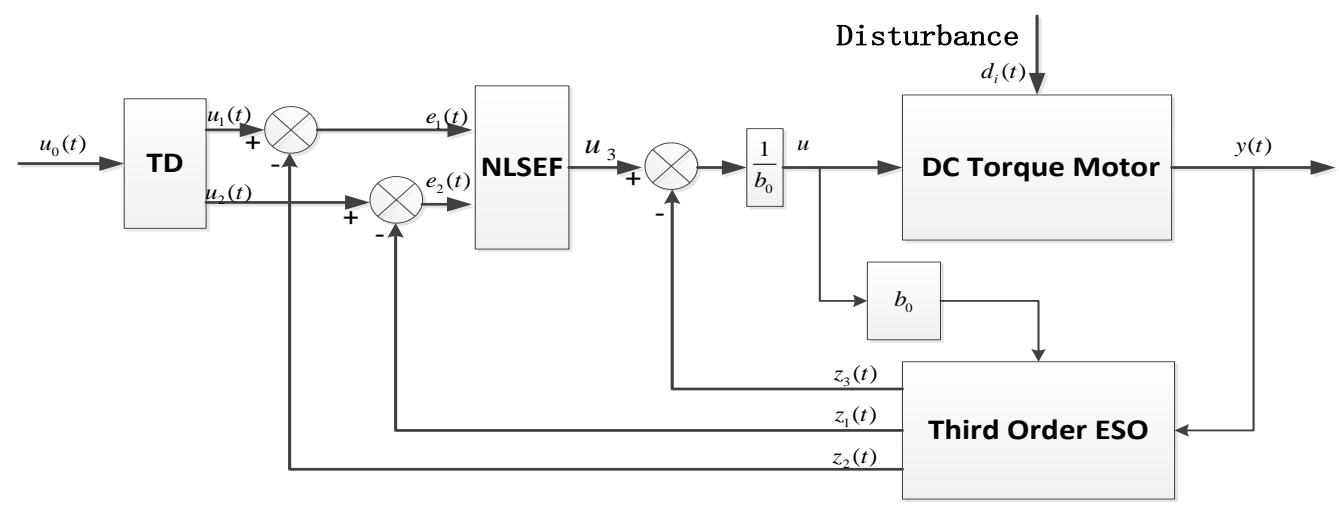

Figure 3. The Structure of Second order ADRC

Tracking Differentiator (TD) form

$\left\{\begin{array}{l}e=u_{1}-u_{0} \\ \dot{u}_{1}=u_{2} \\ u_{0}=\operatorname{fhan}\left(u_{1}, u_{2}, r, h\right)\end{array}\right.$

Where ${ }^{u_{1}}$ is used for tracking input variable ${ }^{u_{0}}, u_{2}$ are used for extracting differential signal from $^{u_{0}}$. The rapid factor of $\operatorname{TD}^{r}$ is 3.1, and the filter factor of $\operatorname{TD}^{h}$ is 0.001 .

In the Extended State Observer (ESO), the state variable of controlled member ${ }^{z_{1}},{ }_{2}$ and the total disturbance ${ }^{z_{3}}$ are both estimated based on the output of controlled member $y$ and the input control signal $u$. The calculation formula of ESO is given as

$$
\left\{\begin{array}{l}
e=y-z_{1} \\
\dot{z}_{1}=z_{2}-\beta_{1} e \\
\dot{z}_{2}=z_{3}+\beta_{2}|e|^{\frac{1}{2}} \operatorname{sign}(e)+b_{0} u \\
\dot{z}_{3}=\beta_{3}|e|^{\frac{1}{4}} \operatorname{sign}(e)
\end{array}\right.
$$

Where the parameter of ESO- $\beta_{1}$ is 16000 ; the parameter of ESO- $\beta_{2} 800000$; the compensatory coefficient $-b_{0}$ is 2 .

Nonlinear Feedback Rate of Dynamic Errors form

$$
\begin{aligned}
& \left\{\begin{array}{l}
e_{1}=u_{1}-z_{2} \\
e_{2}=u_{2}-z_{1} \\
u_{0}=\beta_{1} \cdot \operatorname{fal}\left(e_{1}, \alpha_{1}, \delta_{1}\right)+\beta_{2} \cdot \operatorname{fal}\left(e_{2}, \alpha_{2}, \delta_{2}\right)
\end{array}\right. \\
& \text { ( } 1
\end{aligned}
$$

Where the System State Error are $e_{1}=u_{1}-z_{2} ; e_{2}=u_{2}-z_{1}$; the Proportional Gain $\beta_{1}=40$; the

Differentia Gain ${ }^{\beta_{2}}=0.8$, and other parameters ${ }^{\alpha_{1}}=0.5 ; \quad \alpha_{2}=0.25 ; \quad \delta_{1}={ }_{2}=0.05$, the Control Rate is $u_{0}=\beta_{1} \cdot \operatorname{fal}\left(e_{1}, \alpha_{1}, \delta_{1}\right)+\beta_{2} \cdot \operatorname{fal}\left(e_{2}, \alpha_{2}, \delta_{2}\right)$. 


\section{Controlled Quantity}

$u=u_{3}-\frac{z_{3}}{b_{0}}$

(

Where the error feedback controlled quantity- $u$ is decided by the estimated value of disturbance- $z_{3}$.

In this paper, the Active Disturbance Rejection Controller can be realized by DSP and the control performance of Control System is improved obviously. These advantages of Active Disturbance Rejection Control Technique are revealed obviously in the severe environment.

\section{Overall Design of the System}

The data processing unit of optoelectronic pod control system acquires control parameters from host computer, modifies inner controls parameters, performs control algorithm through acquiring all sensors data and implement control actions of corresponding mode.

The hardware structure of optoelectronic pod control system, which is shown in Figure 4, consists mainly of power supply circuit, drive current detection circuit(the drive current of DC torque motor), analog to digital conversion circuit, communication interface circuit, control parameters storage circuit, digital signal processing circuit and DC motor driver circuit. The hardware systems are integrated in one PCB.

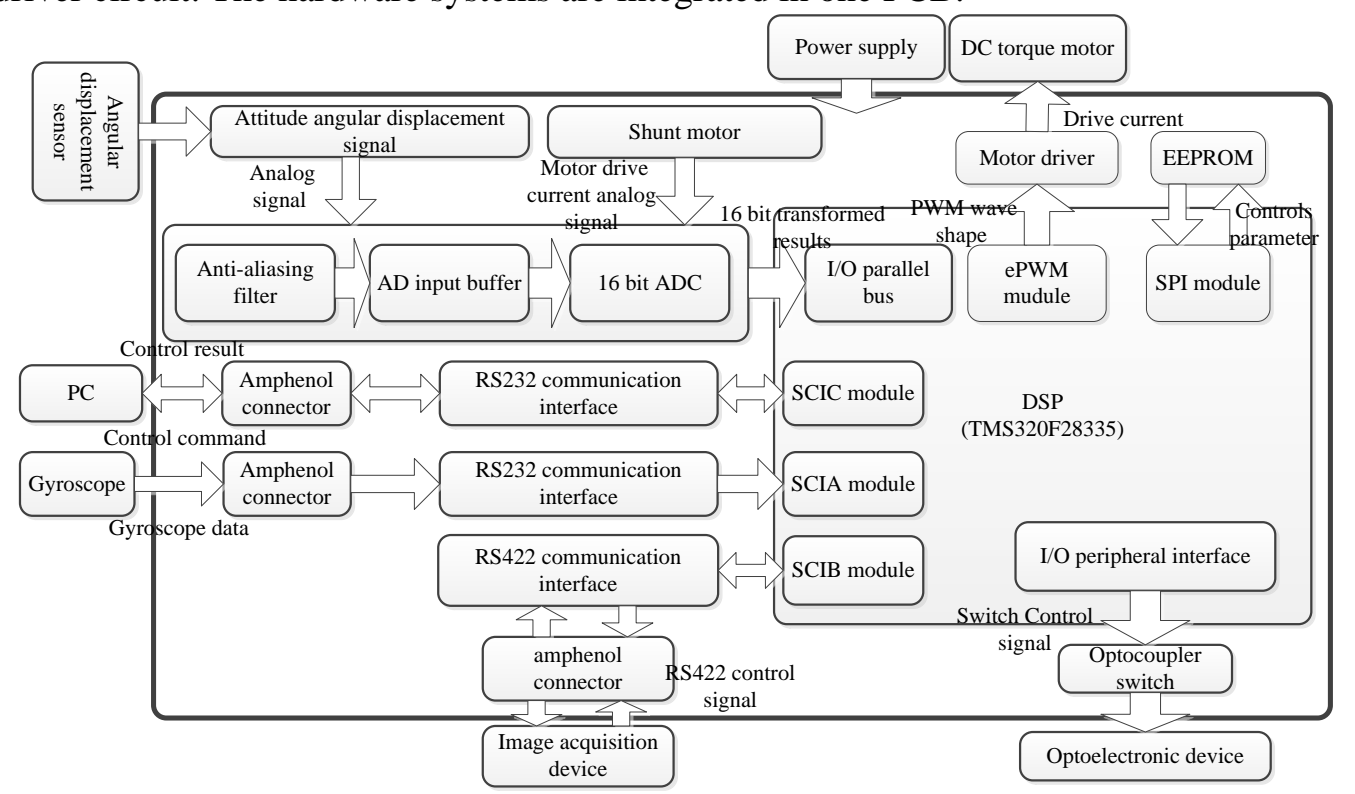

Figure 4. Schematic Architecture of the Optoelectronic Pod Control System

Power supply circuit mainly provides accurate power for the core DSP and its peripheral chips. Meanwhile, it provides power for all chips on the hardware system to ensure that the control system can operate stably. Sensor signal acquisition circuit mainly realizes the following functions: analog signal filtering, signal sampling, signal holding, and high-precision analog-digital conversion.

The core of the digital signal processing circuit is DSP 28335. The DSP acquires control mode command from host computer by its SCI module. Then, DSP reads control parameters of corresponding with its SPI module and then modifies control parameters of control algorithm by itself. After algorithm flag bit is set, DSP outputs PWM waveform with inner PWM modules as control results and then controls motor driver chip to drive DC torque motors. In order to get better control effects, motor shunt detects the current of 
DC torque motors in real time, then the detection signal feedback to DSP and achieves loop control with DC torque motors. Due to actuator, temperature protection was added to protect actuator from damaged by higher temperature.

\section{Hardware Design of the System}

The following will introduce the power supply circuit, sensor signal acquisition circuit, data processing circuit, communication interface circuit, control parameters storage circuit, actuator driving circuit respectively in detail.

\subsection{Power Supply Circuit Design}

According to DSP320F28335 adopts double power supply mode [6], the mode is that power supply of DSP core $(1.9 \mathrm{~V})$ and its peripheral voltage are segregated, as to decrease power dissipation of the chip on running. The TPS62040DGQ-step down converter supplies for the core of DSP and it can output 1.9V and its load current can reach 1.2A with up to $95 \%$ conversion efficiency. The chips were integrated in thermal shutdown circuit and short-circuit protection circuit to prevent damage from incorrectly operating. The schematic circuit diagram as shown in figure 5.

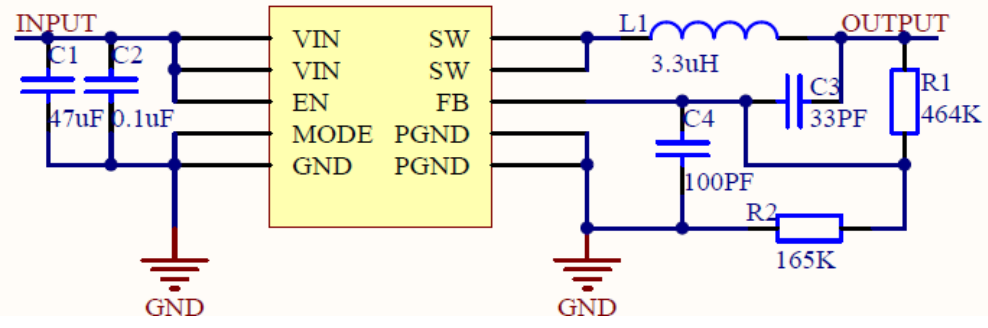

Figure 5. Schematic Circuit Diagram of TPS62040DGQ

Because of the nature of the buck converter has pulsating input current, a low ESR input capacitor is required. The input capacitors should be connected $47 \mathrm{uf}(\mathrm{C} 1)$ with $0.1 \mathrm{uf}$ (C2) ceramic capacitor parallel to decrease external disturbance. The chip typically uses 3.3uh (L1) output inductor with the lowest DC resistance for high efficiency. The output terminal of chip uses small ceramic capacitors to decrease ripple waves.

DSP's core-voltage controls its peripheral voltage with triode switch circuit. For instance, if DSP core voltage is 0, DSP peripheral power supply output is 0 , avoided destroying DSP with voltage surge. TPS75733KKT low-dropout voltage regulator outputs $3.3 \mathrm{v}$ voltage and $3 \mathrm{~A}$ load current. The chip was inner integrated in thermal shutdown protection circuit and its load regulation is merely 3\%. Because of large transient currents will cause the input voltage to droop. If this droop causes the input voltage drop below the UVLO threshold, the device will turn off. The 47uf (C5) bypass capacitor of the input end is added to ensure device stability. The TPS75733KKT requires a filter capacitor (C7, C8) connected between OUT and GND to stabilize the internal control loop. The schematic circuit diagram as shown in figure 6.

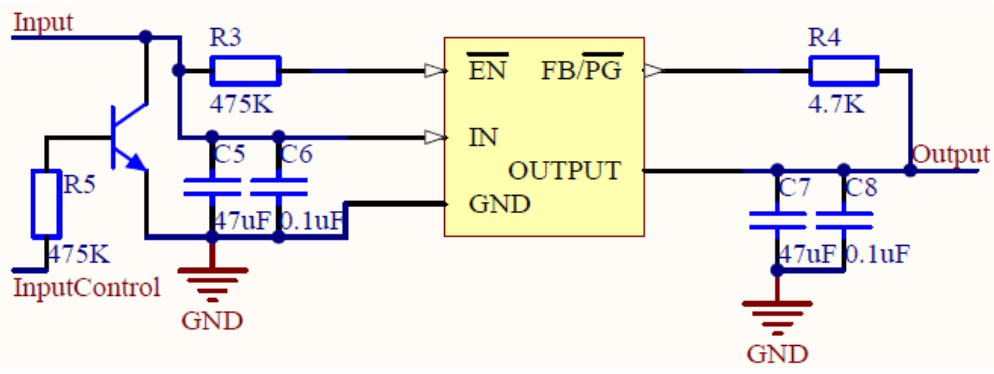

Figure 6. Schematic Circuit Diagram of TPS75733KKT 


\subsection{Drive Current Detection Circuit Design}

In the process of DC torque motor driver working, motor shunt (Shunt-R) detects the drive current of DC torque motor, in this paper, the $0.1 \Omega$ resistance was selected as motor shunt. As the detected signal is feeble, current amplifier unit of the circuit was added to realize amplification of current signal. In order to convert the current signal by analog-digital converter, current-voltage convertor unit of the circuit was added to ensure that ADC (analog to digital converter) can convert the signal of motor drive current. The drive current detection circuit was designed as figure 7, AD8210YRZ-current shunt monitor was chosen to achieve these requirements.

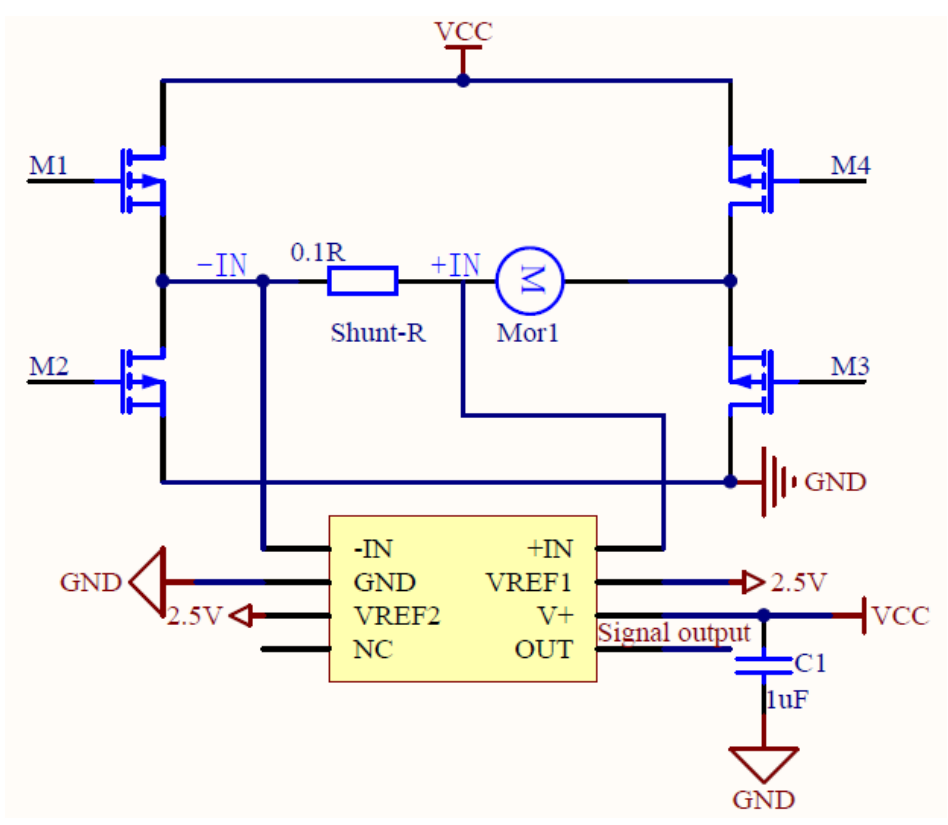

Figure 7. Schematic Circuit Diagram of AD8210YRZ

The chip has high common-mode voltage range, high bandwidth and its open loop gain can reach 20 times. The schematic circuit was shown in figure 7 , the non-inverting terminal of the chip connect with front-end shunt resistor (-IN Point), and inverting terminal connect with rear-end shunt resistor ( $+\mathrm{IN}$ Point). The fluctuation of output voltage is based on reference voltage $(2.5 \mathrm{~V})$.

\subsection{Analog-Digital Conversion Circuit Design}

The circuit needs to transform four channels sensor signals into 16bit digital data, and then transfers digital data to DSP immediately though 16bit parallel bus [7]. The circuit was designed as figure 8 , AD7606BSZ analog digital converter was selected to achieve those requirements. 


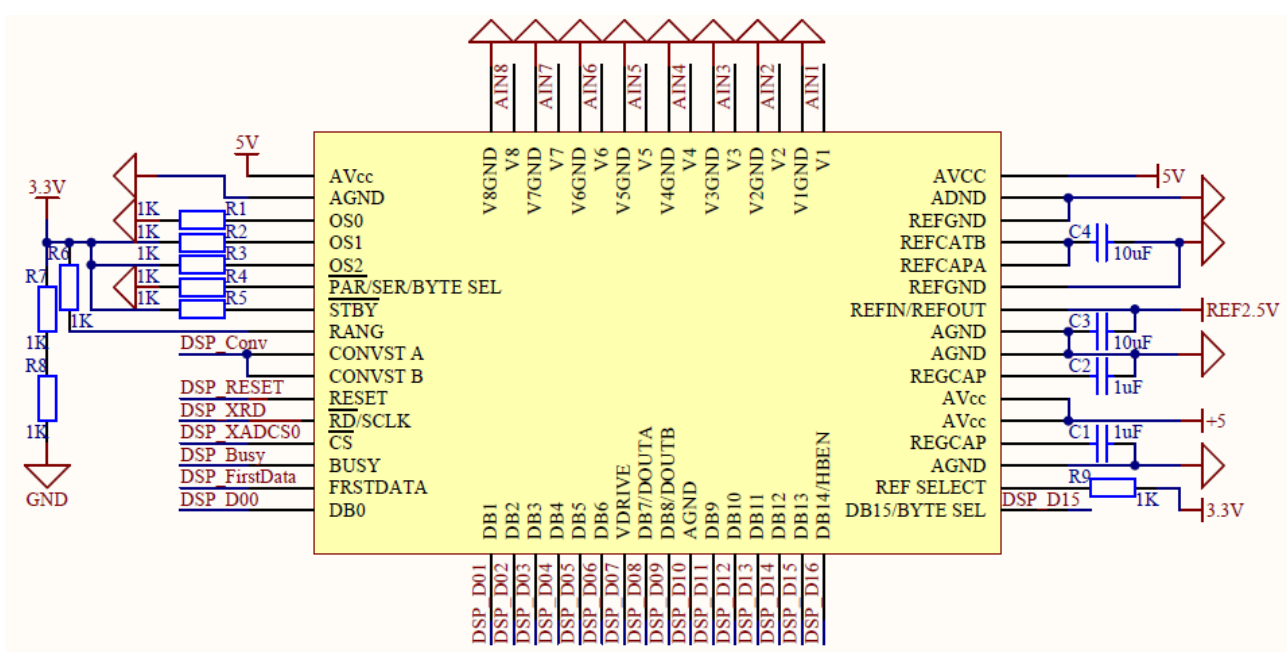

Figure 8. Schematic Circuit Diagram of AD7606BSTZ

The AD7606BSZ analog-digital converter has 8 conversion channels with 16 bit in the chip, it can be selected two output modes (parallel bus, serial port). After AD7606BSZ converted 4 channels signals in sequence, DSP acquires digital data by ADC peripheral interrupt.

\subsection{Communication Interface Circuit Design}

Because of the SCI modules has difference between host computer serial ports in logic and voltage level [8]. The communication interface circuit realizes transformation between SCI module of DSP and host computer, and ensure that DSP can maintain communication steadily with host computer. The circuit was designed as figure 9, MAX3232 and MAX3488EESA were selected to achieve these requirements.

The MAX3232 has 2 receivers and 2 drivers, and can run at data rates of 120kps while maintaining RS232 output levels. The schematic circuit was shown in figure 6-7, the chip charge pump requires a flying capacitor $(\mathrm{C} 10, \mathrm{C} 11)$ and a reservoir capacitor $(\mathrm{C} 12, \mathrm{C} 13)$ to generate the $\mathrm{V}+$ and $\mathrm{V}$ - supplies.

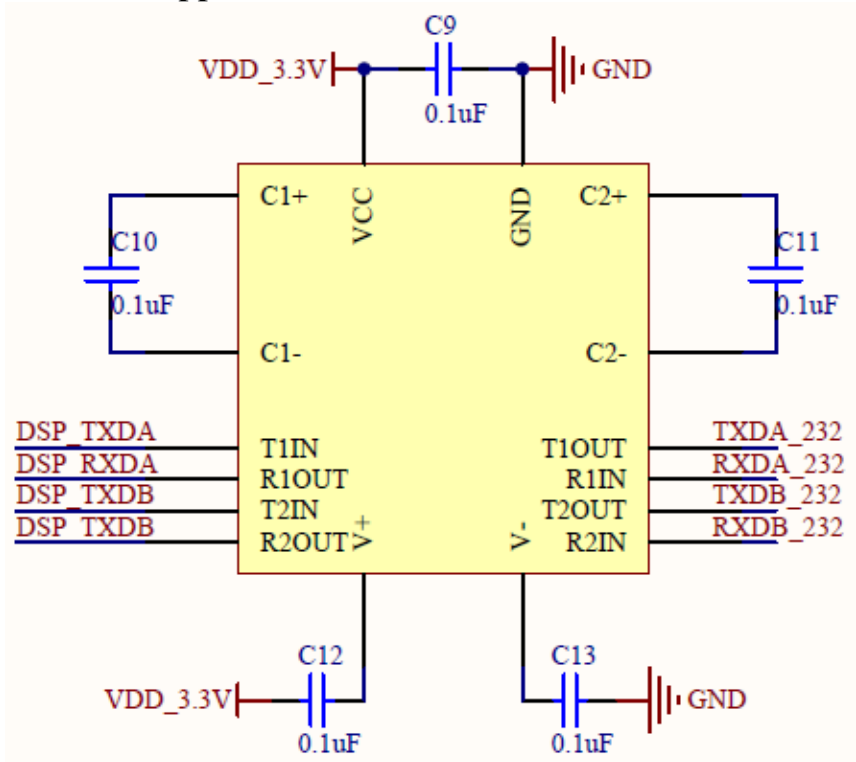

Figure 9. Schematic Circuit Diagram of MAX3232 
The MAX3488EESA contains one driver and one receiver, shown in figure 10, the chip is selected to focus vision facilities and the matched resistances (R6, R7) in the output terminal and the output terminal are added to ensure the signal not to be distorted.

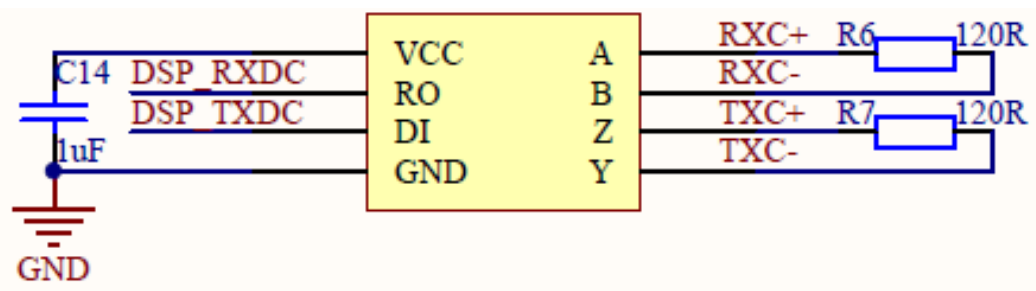

Figure 10. Schematic Circuit Diagram of MAX3488EESA

\subsection{Torque Motor Drive Circuit Design}

In this paper, DC torque motor drive circuit is used to control DC torque motor in direction of rotation and rotational speed [8]. Meanwhile, the circuit monitors the drive current of motor in real time and sends the current signal to DSP as to implement the loop control of DC torque motor. The circuit was designed as figure 11, DRV8412 was selected to achieve these requirements.

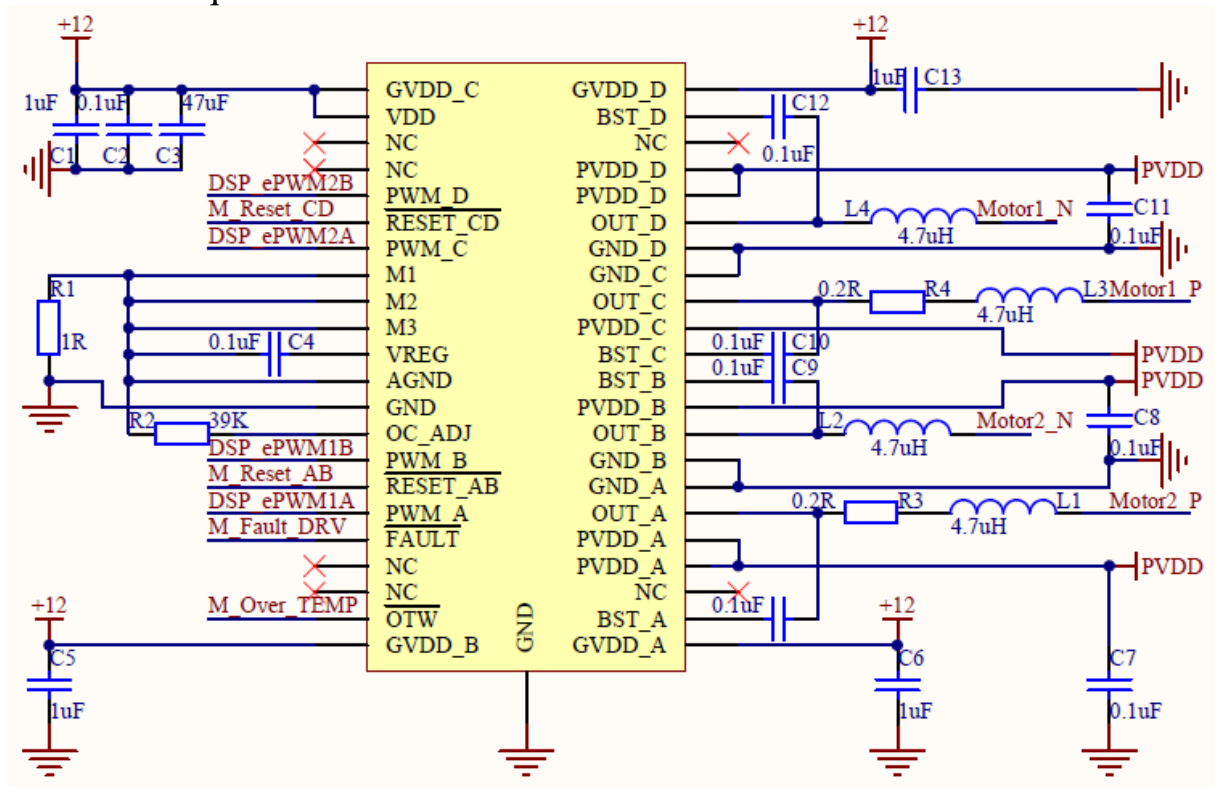

Figure 11. The Schematic Circuit Diagram of DRV8412

The DRV8412-dual full bridge PWM motor driver has two channels of output terminals and it can change direction of rotation and revolving speed of motor with phase separation and duty cycle. The chip is integrated with temperature detecting circuit inner to monitor the inner temperature of chip in real time, and take correspond action by judging whether beyond the threshold or not, and then outputs fault results. The schematic circuit as shown in figure11, PWM input terminals of the chip are connected with output terminals of DSP ePWM modules to control driver output. Fault signal pin connects with a peripheral interrupt pin to transmit fault signal to DSP. In order to provide low di/dt output and proper protection during overload condition, output inductors (L1, L2, L3, L4) are added during normal operation. 


\subsection{Control Parameters Storage Circuit Design}

The system needs different groups of control parameters to test this control system in good condition or not, in the process of debug. In order to get better control result, DSP assembles different control parameters into new groups through reading storage circuit. After debug, the circuit stores different mode control parameters [9] and DSP can read these parameters depend on different control modes to realize self-modifying of control algorithm. The circuit was designed as figure 12, M95M01-R was selected to achieve these requirements.

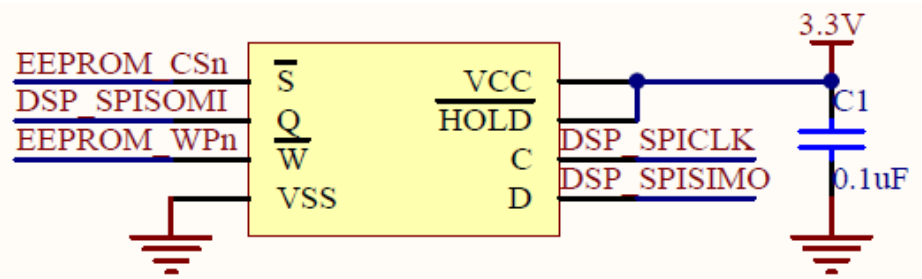

Figure 12. Schematic Circuit Diagram of M95M01-RMN6

The M95M01-R is an electrically erasable programmable memory device. it is accessed by a high speed SPI-compatible bus, and the memory array is organized as $131072 \times 8$ bit.

\subsection{TMS320F28335 Data Processing Circuit Design}

TMS320F28335 data processing circuit [10] mainly realizes the following functions: data acquisition, communication with host computer, execution of control algorithm, and the output of the control waveforms. Host computer send control commands to DSP by the communication interface, and the control commands including mode commands, joystick rotation angle and etc. DSP switches to relevant control algorithm and modifies control parameters through reading EEPROM. DSP acquires gyroscope data by inner SCIA (serial communication interface A) module [11], and gets detected data though peripheral bus. The inner SCIC (serial communication interface C) module mainly in charge of communication with host computer, and taking advantage of high speed computing power of DSP28335, control algorithm can be quickly executed. The DSP28335 of ePWM modules output PWM waveform after performing control algorithm to control drive unit and achieves optoelectronic pod rotation control.

\section{System Software Design}

Taking full account of the hardware design, software of the DSP28335 is carried out. Software process of DSP28335 is shown in figure 13, SCIC interrupt is responsible for receiving host computer's command data, checking command data frames and converting command data and then DSP sets control algorithm based on command of host computer. Then, DSP28335 reads control parameters from EEPROM through SPI bus and modifies algorithm parameters by itself. ADC interrupt is responsible for receiving digital data by analog-digital converter, then converting data by accuracy conversion formula and then the converted data were send to algorithm as control input to process. The output of control results are depend on inquiring algorithm ending flag, if the flag was not be set ,the control algorithm would continue. After the ending flag is set, the control results are outputted. 


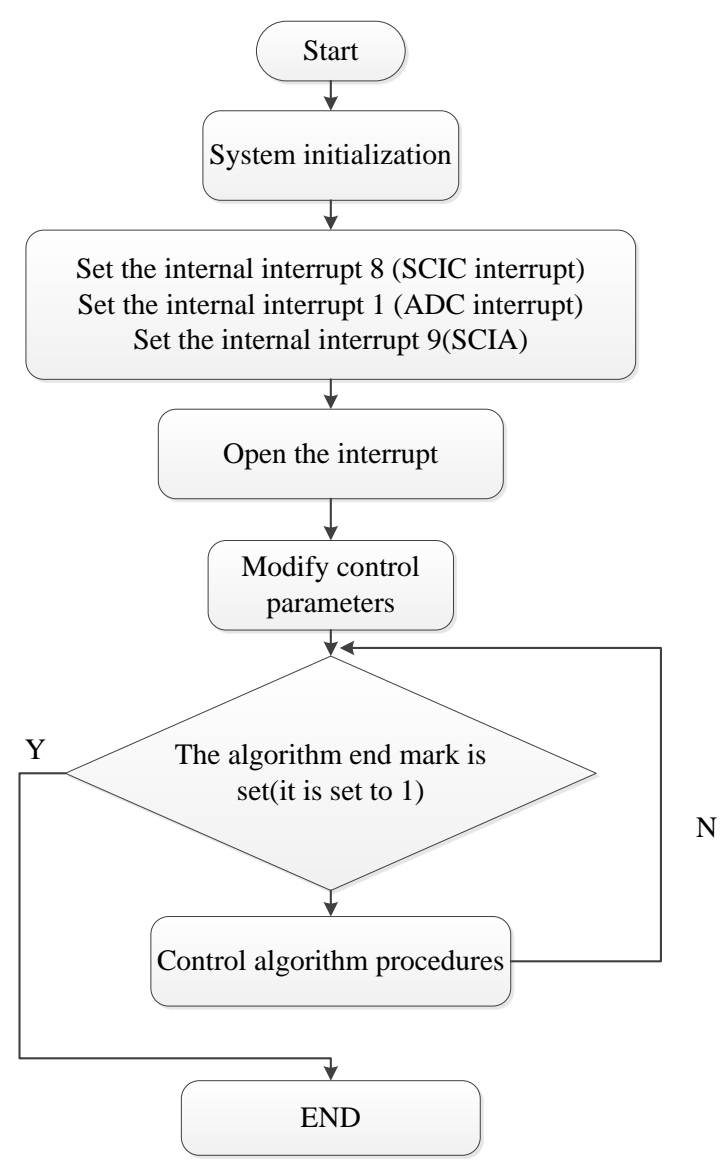

(a)DSP28335 Main Program Flowchart

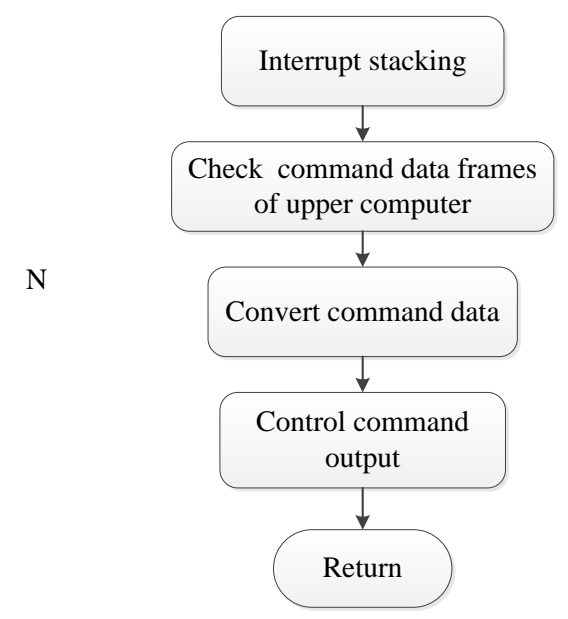

(b) SCIC Serial Port Interrupt

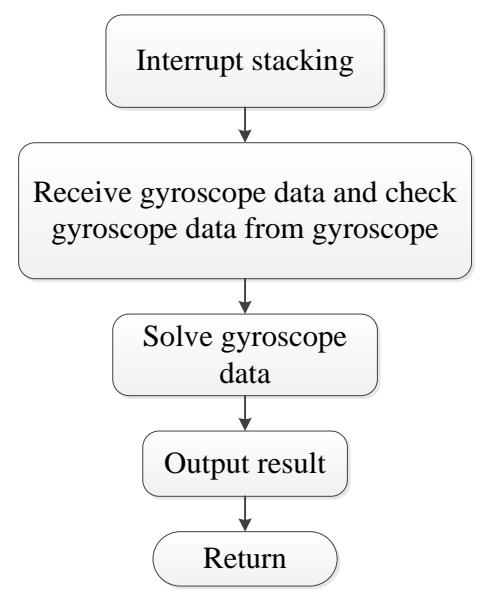

(c) Gyroscope Serial Port Interrupt

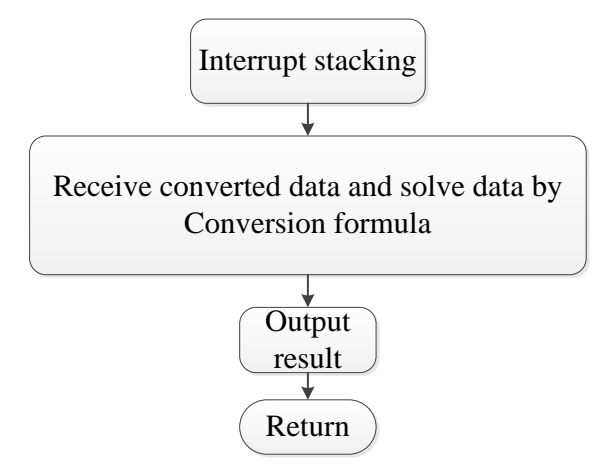

(d) ADC Interrupt 


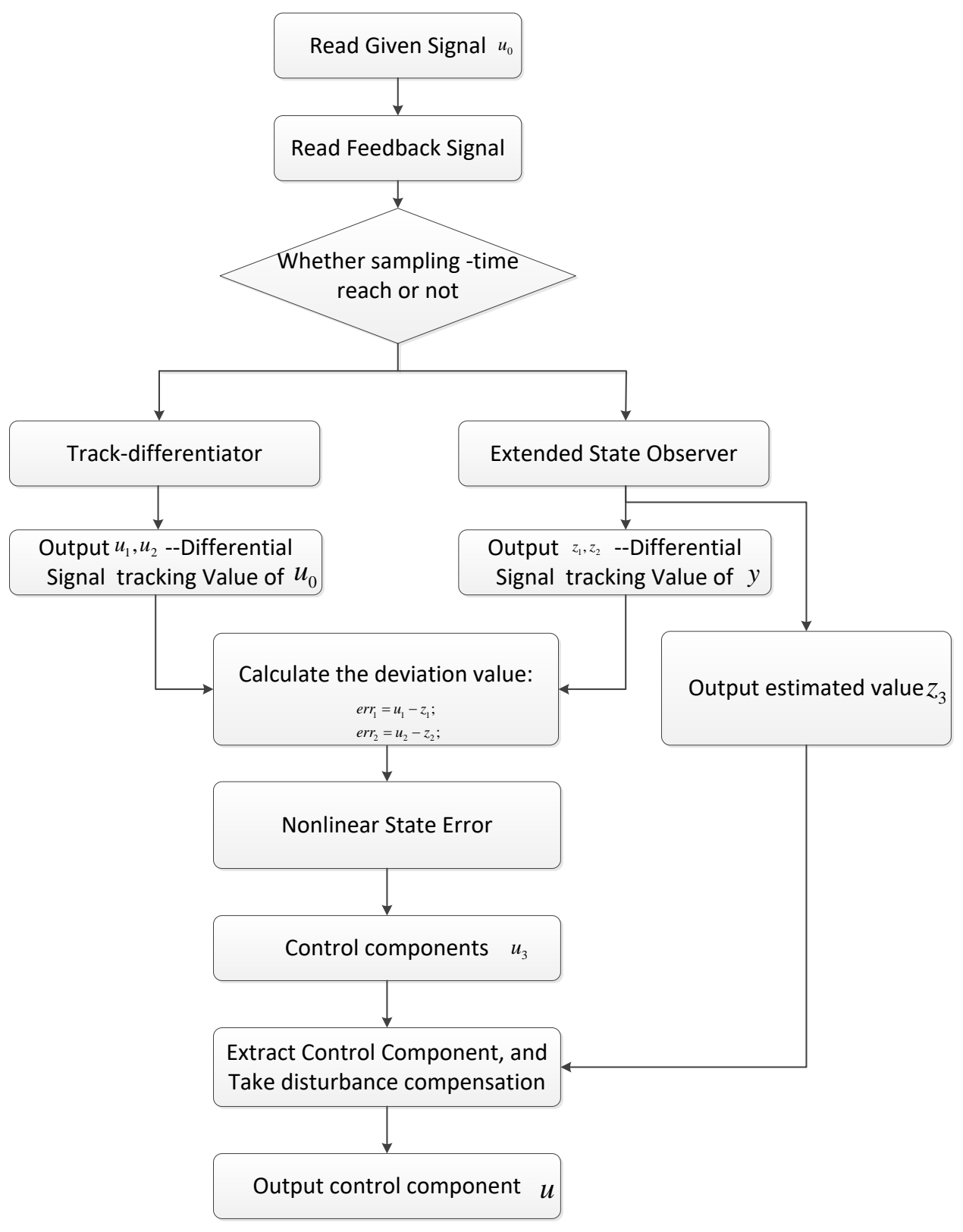

(e) Active Disturbance Rejection Control Algorithm

Figure 13. DSP28335 Program Flow Chart

\section{Experiment and Results}

Figure 14shows the hardware platform for Optoelectronic pod control system, including power supply unit, communication interface unit, analog-digital conversion unit, control parameters storage circuit, DSP master control circuit, and equipment control circuit. As shown in Figure 15, in the bottom of hardware platform, some interfaces were designed to ensure the hardware platform can be installed into the inner pod, these interfaces including that sensor interface, gyroscope data interface, motor drive interface, $28 \mathrm{~V}$ DC power interface, $5 \mathrm{~V}$ DC power interface. The figure 16 is installation effect map of hardware platform. 


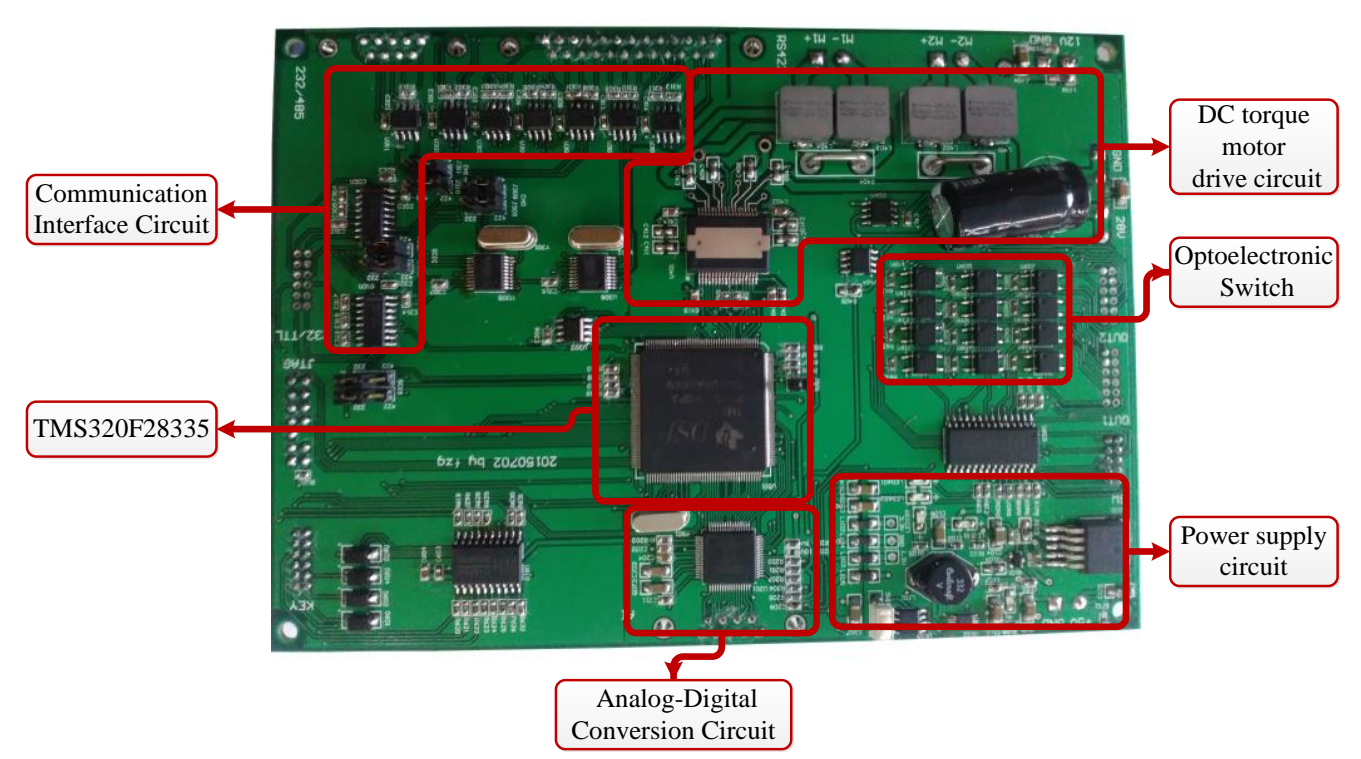

Figure 14 Photo of the top layer of Optoelectronic pod Control Hardware System

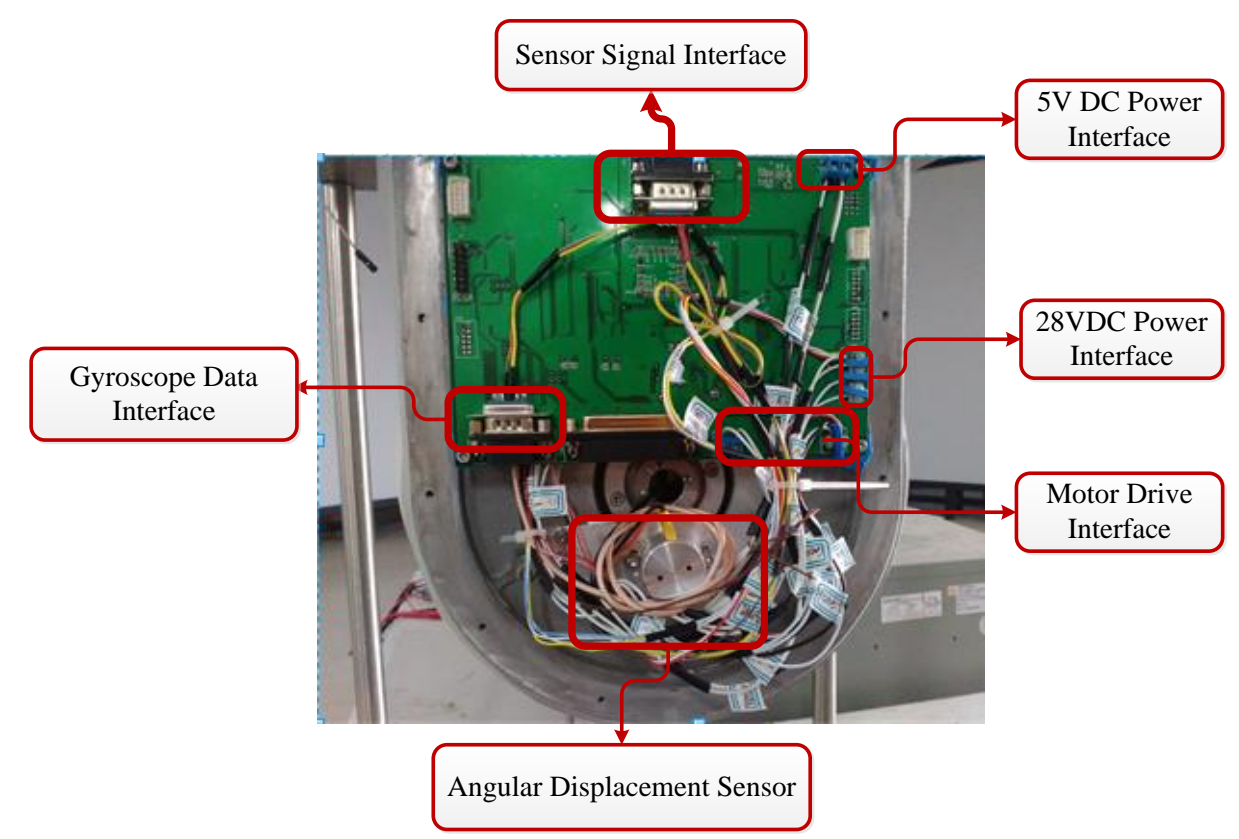

Figure 15. Photo of the Bottom Layer of Optoelectronic pod Control Hardware System 


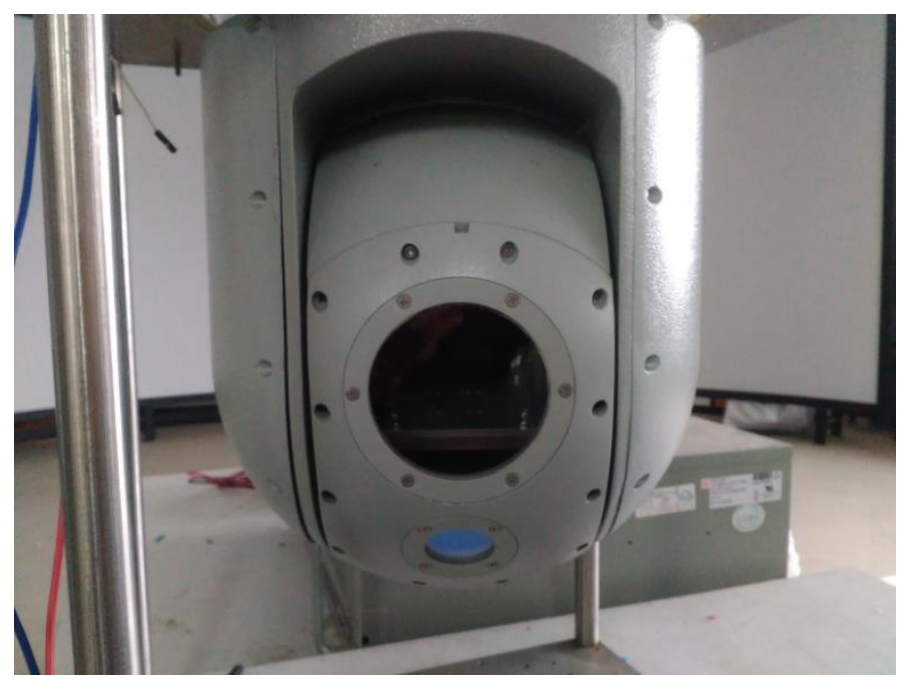

Figure 16. Photo of Optoelectronic pod Control System

The experiment was accomplished on the airborne electro-optoelectronic pod, host computer send control command of tracking mode to DSP, and then electro-optoelectronic pod can immediately responded to the command through driving the motors in pitching and yaw directions by DSP. In the process of executing, the figure 17 illustrates the following respond in the direction of yaw with constant input angular. The figure 18 illustrates the following respond in the pitching direction with constant input angular. From these three pictures, we can see that, system responds quickly with a little overshoot and no large static errors were appeared with rotational inertia. In addition, in the servo mode, the airborne electro-optical can respond control command in real time. The DSP can quickly adjust the imaging equipment focus which based on host computer commands.

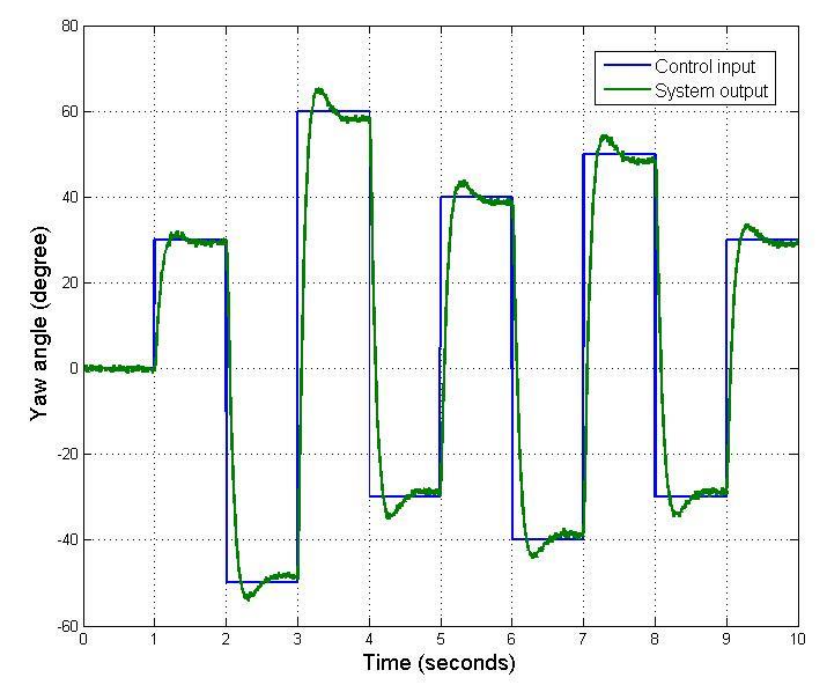

Figure 17. System Following Response in the Yaw Direction 


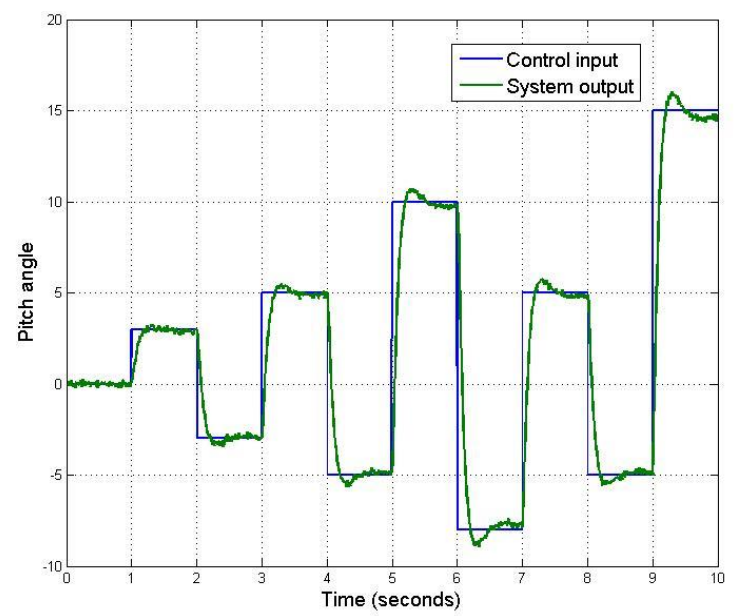

Figure 18. System Following Response in the Pitching Direction

According to Test Standard for Military Equipment, the major vibration of plane is Fixed Frequency Vibration. In order to test the stabilization precision of optoelectronic pod, the optoelectronic pod was installed on the Platform vibrator to simulate Vibration performances, the speed of Pitch and the speed of Yaw both were detected by the gyroscope, the test results of the Stabilization Precision by stratified sampling as Table 2:

Table 2. The Test Result Statistical List of the Stability Accuracy

\begin{tabular}{|c|c|c|c|c|c|}
\hline \multicolumn{2}{|c|}{ Vibration Condition } & & \multicolumn{3}{|c|}{ Stabilization Precision ( urad ) } \\
\hline $\begin{array}{c}\text { Frequency } \\
(\mathrm{Hz})\end{array}$ & $\begin{array}{l}\text { Amplitude } \\
(\mathrm{g})\end{array}$ & & $\mathrm{X}$ to Vibration & Y to Vibration & $\mathrm{Z}$ to Vibration \\
\hline \multirow{2}{*}{3} & \multirow{2}{*}{0.2} & Pitch & 113.33 & 105.57 & 17.26 \\
\hline & & Yaw & 25.40 & 25.73 & 7.89 \\
\hline \multirow{2}{*}{5} & \multirow{2}{*}{0.15} & Pitch & 113.40 & 106.61 & 4.25 \\
\hline & & Yaw & 25.23 & 26.75 & 11.60 \\
\hline \multirow{2}{*}{10} & \multirow{2}{*}{0.1} & Pitch & 141.88 & 148.46 & 15.31 \\
\hline & & Yaw & 26.02 & 127.06 & 10.18 \\
\hline \multirow{2}{*}{15} & \multirow{2}{*}{0.1} & Pitch & 113.82 & 104.86 & 9.37 \\
\hline & & Yaw & 26.5 & 34.45 & 14.61 \\
\hline \multirow{2}{*}{20} & \multirow{2}{*}{0.1} & Pitch & 108.01 & 102.83 & 30.29 \\
\hline & & Yaw & 24.61 & 24.66 & 43.12 \\
\hline \multirow{2}{*}{25} & \multirow{2}{*}{1.0} & Pitch & 189.45 & 165.19 & 71.37 \\
\hline & & Yaw & 40.00 & 162.05 & 31.64 \\
\hline \multirow{2}{*}{51} & \multirow{2}{*}{0.6} & Pitch & 106.57 & 5.54 & 9.56 \\
\hline & & Yaw & 24.83 & 32.52 & 17.30 \\
\hline \multirow{2}{*}{76} & \multirow{2}{*}{0.6} & Pitch & 106.74 & 5.88 & 5.63 \\
\hline & & Yaw & 26.66 & 24.54 & 17.81 \\
\hline \multirow{2}{*}{102} & \multirow{2}{*}{0.6} & Pitch & 134.87 & 10.74 & 68.65 \\
\hline & & Yaw & 31.92 & 30.65 & 24.42 \\
\hline
\end{tabular}

\section{Conclusions}

In this paper, an optoelectronic pod hardware system based on DSP TMS320F28335 is described. The hardware design and software design are described in detail. The experiment results show that: DSP data processing unit can acquire sensor data correctly, 
communication interfaces can work properly, and control algorithm can perform correctly in the vibration environment and it can compensate perturbance immediately. According to the test results of the Stabilization Precision on the vibro-bench, the Stabilization Precision of optoelectronic pod is able to achieve $30 \mathrm{urad}$ that can meet absolutely controlled requirements. All of vibrational conditions were both simulate extreme vibrational environments in the vibration testing process, so we can see that the ADRC control algorithm performed on the DSP can effectively retrain vibrational disturbance. Meanwhile, ADRC control algorithm not obviously effects the program execution speed of DSP. In the servo mode, host computer send control command of tracking mode to DSP, and then electro-optoelectronic pod can immediately responded to the command through driving the motors in pitching and yaw directions by DSP. From the servo mode test we can see that system responds quickly with a little overshoot and no large static errors were appeared with rotational inertia. In addition, in the servo mode, the airborne electro-optical can respond control command in real time. The DSP can quickly adjust the imaging equipment focus which based on host computer commands. It can quickly adjust the imaging equipment focus which based on host computer commands.

\section{Acknowledgements}

The authors would like to thank the financial support of the financial support of the 2014 high school talent support plan second levels of Liao Ning province China. LJQ2014017.

\section{References}

[1] Z. Yu, J.-Z. Cao, H.-T. Yang, H.-N. Guo, B. Gao and L. Yang, "Advanced fuzzy PID composite control for stabilized platform system", International Conference on Mechatronics and Automation, (2012); Chengdu, China.

[2] J.-D. Shang, D.-F. Hu and L.-B. Wang, "Fuzzy-PID control for control system of airbone optoelectronic pod", 2nd Annual Conference on Electrical and Control Engineering, (2011); Yichang, China.

[3] Y.-Q. Wei, J.-N. Xu and T. Zhu, "Fuzzy control in the DC motor servo system for gravimeter stabilized platform", International Conference on Electrical Machines and Systems, (2013); Busan, Korea.

[4] P. Wang, G.-Y. Zhang, J.-Y. Liu, Y.-J. Gao and M. Huang, "Irrotational displacement vibration isolation on optoelectronic pod", Chinese Society of Astronautics, vol. 41, no. 10, (2012), pp. 2799-2804.

[5] X.B. Wang, J. Liu, D.-L. Yang and K. Wang, "On ADRC for photoelectrical stabilized platform", 2nd International Conference on Mechanic Automation and Control Engineering, (2011); Hohhot, China.

[6] B. Tamyurek, A. Ceyhan, E. Birdane and F.Keles, "A simple DSP based control system design for a three-phase high power factor boost rectifier", 23rd Annual IEEE Applied Power Electronics Conference and Exposition, (2008); TX, United states.

[7] J. Baum and E. Jacobsen, "Very-low-pressure smart sensing with serial communications interface", Helmers Publ Inc, vol. 13, no. 1, (1996), pp. 27-34.

[8] H. K. S. Ransara and U. K. Madawala, "A Torque Ripple Compensation Technique for a Low-Cost Brushless DC Motor Drive”, Institute of Electrical and Electronics Engineers Inc, vol. 62, no. 10, (2015), pp. 6171-6182.

[9] D. Bursky, "Flash and EEPROM technologies combine on feature-rich MCUs", Penton Publ Inc, vol. 45, no. 11, (1997), pp. 81-82, 84, 86, 88, 90, 92, 94.

[10] G. V. Kshirsagar, G. N. Mulay and S. Yeolekar, "TMS320F28335 based single phase overcurrent protection Implementation using Numerical Relay”, 6th IEEE Power India International Conference, PIICON, (2014).

[11] T.-Q. Xu, W. Xu and Y.-J. Piao, "Design of gyroscope signed acquisition system in space instrument based on L3G400D", 3rd International Conference on Instrumentation and Measurement, Computer, Communication and Control, (2013); Shenyang, Liaoning, China, (2013). 


\section{Authors}
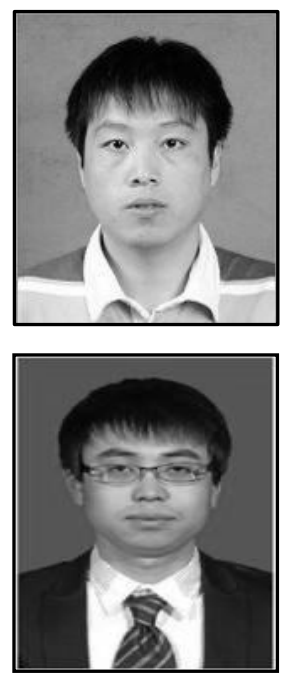

Zhigang Feng, $\mathrm{He}$ is an associate professor of Shenyang Aerospace University. He received his Doctor's Degree from Harbin Institute of Technology, P.R. China at 2009. His main research direction includes system fault diagnosis, self-validating sensor and self-validating actuator.

Ming Jin, he received the bachelor degree of Measuring and Control Technology and Instrumentations Automatization from Shenyang Aerospace University, China, in 2013. He is currently study for a master degree in the School of Automation, Shenyang Aerospace University. 